\title{
Uma contribuição à saúde dos trabalhadores: um guia sobre exposição aos fluídos biológicos
}

\author{
A CONTRIBUTION TO OCCUPATIONAL HEALTH: A GUIDE ON THE EXPOSURE TO \\ BIOLOGICAL FLUIDS
}

\section{UNA CONTRIBUCIÓN A LA SALUD DE LOS TRABAJADORES: GUÍA SOBRE EXPOSICIÓN A LOS FLUIDOS BIOLÓGICOS}

\section{Fernanda Moura D’Almeida Miranda', Altair Von Stein Junior ${ }^{2}$, Silvia Petreli ${ }^{3}$, Moacir Ramos Pires ${ }^{4}$, Leticia Gramazio Soares ${ }^{5}$, Bárbara Nicolato Ribeiral ${ }^{6}$, Leila Maria Mansano Sarquis ${ }^{7}$, Vanda Elisa Andres Felli ${ }^{8}$, Marina Ciola Lorusso Xavier de Oliveira ${ }^{9}$}

\section{RESUMO}

Os trabalhadores da saúde estão expostos a riscos ocupacionais no seu ambiente de trabalho, destacando-se, dentre eles, o risco biológico. A conduta esperada do trabalhador após esta exposição deve ser definida e orientada, para que medidas preventivas possam ser adotadas. Assim, pretende-se com este guia fornecer subsídios para a conduta dos trabalhadores em relação à exposição biológica, contribuindo para a adesão ao protocolo de monitoramento.

\section{DESCRITORES}

Riscos ocupacionais

Enfermagem

Pessoal de saúde

Saúde do trabalhador

\begin{abstract}
Health workers are exposed to occupational hazards at the workplace, among which we highlight the biological risk. Information and orientation should be provided regarding the conduct that workers are expected to follow in the case becoming exposed. Thus, this guide is intended to provide the elements to orient workers' conduct in cases of biological exposure, improving adherence to the monitoring protocol.
\end{abstract}

\section{DESCRIPTORS \\ Occupational risks \\ Nursing \\ Health personnel \\ Occupational health}

\author{
RESUMEN \\ DESCRIPTORES \\ Riesgos laborales \\ Enfermería \\ Personal de salud \\ Salud ocupacional
}

Los trabajadores de la salud están expuestos a riesgos ocupacionales en su ambiente de trabajo, entre ellos se destaca el riesgo biológico. La conducta esperada del trabajador luego de esta exposición debe ser definida y orientada para que medidas preventivas pueden ser adoptadas. De este modo, se pretende con esta guía brindar ayuda a la conducta de los trabajadores en relación a la exposición biológica, contribuyendo a la adhesión al protocolo de monitoreo.

\footnotetext{
${ }^{1}$ Mestranda em Enfermagem da Universidade Federal do Paraná. Especialista em Epidemiologia e Controle de Infecção Hospitalar. Enfermeira da Unidade Saúde do Trabalhador. Membro do Grupo de Pesquisa GEMSA. Curitiba, PR, Brasil. fernandadalmeida79@hotmail.com ${ }^{2}$ Mestre em Tecnologia em Saúde. Enfermeiro da Unidade Saúde do Trabalhador. Curitiba, PR, Brasil. altairstein@pop.com.br ${ }^{3}$ Especialista em Treinamento de Recursos Humanos. Assistente Social da Unidade Saúde do Trabalhador. Curitiba, PR, Brasil.silviapetreli@sesa.pr.gov.br ${ }^{4}$ Médico Infectologista da Unidade Saúde do Trabalhador. Curitiba, PR, Brasil. dr.mpramos@gmail.com ${ }^{5}$ Enfermeira. Mestranda da Universidade Federal do Paraná. Curitiba, PR, Brasil. leticiagramazio@yahoo.com.br ${ }^{6}$ Enfermeira pela Universidade Federal do Paraná. Curitiba, PR, Brasil. barniri@hotmail.com ${ }^{7}$ Enfermeira. Professora Adjunta do Curso de Graduação em Enfermagem da Universidade Federal do Paraná. Membro do Grupo de Pesquisa GEMSA. Membro do grupo de Pesquisa de Qualidade de Vida do Departamento de Administração em Enfermagem da Escola de Enfermagem da Universidade de São Paulo. Curitiba, PR, Brasil. leila.sarquis@ufpr.br ${ }^{8}$ Enfermeira. Professora Associada do Departamento de Orientação Profissional da Escola de Enfermagem da Universidade de São Paulo. Líder do Grupo de Pesquisa Estudos sobre a Saúde do Trabalhador de Enfermagem da Escola de Enfermagem da Universidade de São Paulo. São Paulo, SP, Brasil. vanda.felli@pq.cnpq.br ${ }^{9}$ Especialista em Psicologia do Trabalho. Psicóloga da Unidade Saúde do Trabalhador. Curitiba, PR, Brasil. ma_marina26@hotmail.com
} 


\section{INTRODUÇÃO}

A possibilidade de exposição aos fluídos biológicos ocorre entre os trabalhadores de saúde, podendo causar agravos à sua saúde. Estes trabalhadores realizam, direta ou indiretamente, o cuidado aos pacientes, expondo-se aos microrganismos existentes no sangue ou em outros fluídos biológicos, tais como urina, fezes entre outros ${ }^{(1-3)}$.

O risco da exposição está presente em diferentes ambientes de trabalho, como Unidades Básicas de Saúde, hospitais, clínicas, ambulatórios, consultórios odontológicos ou médicos e em serviços de saúde. No entanto, o ambiente hospitalar apresenta maior complexidade, principalmente porque concentra pacientes com doenças infecciosas, infecto-contagiosas e parasitárias e, ainda, porque concentra um grande número de trabalhadores ${ }^{(4-6)}$.

O risco do trabalhador de desenvolver infecções, após exposição a material biológico, é variável e depende do tipo de acidente e de outros fatores. Portanto, estes acidentes são considerados casos de emergência, devido a necessidade da quimioprofilaxia para a prevenção do vírus do Imunodeficiência Humana (HIV) nas primeiras duas horas após exposição biológica. Os ferimentos com agulhas e materiais perfurocortantes são considerados perigosos, pois podem transmitir mais de 20 tipos de patógenos diferentes, entre eles o vírus do HIV, o vírus da Hepatite $B$ e da Hepatite $C$. O risco médio de adquirir o HIV é de aproximadamente 0,3\% após exposição percutânea, e de 0,9\% após a exposição através de mucosas. A probabilidade de infecção pelo vírus da hepatite $B$ após a exposição percutânea é superior à probabilidade de infecção pelo HIV, podendo atingir $40 \%$. Para o vírus da hepatite C, o risco médio é de $1,8 \%{ }^{(7)}$.

Uma maneira de evitar ou diminuir estes acidentes é a utilização das medidas preventivas, com o uso do Equipamento de Proteção Individual (EPI) como luvas, óculos, avental e máscara; o estabelecimento de normas de biossegurança, o não-reencape de agulhas, o descarte adequado de material perfurocortante imediatamente após o uso, a realização e capacitação através de educação continuada e a imunização vacinal|(7).

Essas medidas nem sempre são adotadas, tanto pelas instituições de saúde como pelos trabalhadores, o que implica em grande exposição dos trabalhadores aos agentes biológicos, como vírus, bactérias, parasitas, entre outros.

Estudos realizados tanto no contexto internacional como nacional têm mostrado a problemática do acidente de trabalho com material biológico dentre os trabalhadores de saúde, permitindo apreender a frequência e a gravidade dessa exposição.
Os casos confirmados, nos Estados Unidos, descritos pelo Centers for Disease Control (CDC), somaram até dezembro de 200157 casos de soroconversão após exposição ocupacional e 138 casos suspeitos de soroconversão entre trabalhadores de saúde. O CDC estima que a cada ano ocorram 385 mil ferimentos perfurocortantes entre os trabalhadores de saúde, ou seja, uma média de 1.000 acidentes percutâneos por dia ${ }^{(8-9)}$. Os acidentes com instrumentos perfurocortantes são a principal forma de transmissão de HIV entre os profissionais de saúde, referido por vários autores ${ }^{(10-13)}$.

A OMS afirma que os trabalhadores de saúde sofrem, aproximadamente, 16.000 infecções pelo vírus da Hepatite $C(\mathrm{HCV}), 66.000$ infecções ao Vírus da Hepatite $B$ (HBV), e 1.000 infecções pelo HIV. Estes dados expressam a problemática de saúde desses trabalhadores, causando incapacidades e doenças que podem comprometer a sua qualidade de vida ${ }^{(14)}$.

Os dados registrados pela Occupational Safety and Health Administration (OSHA), no período de 1992 a 2002, dos 67.363 trabalhadores que morreram de doenças ocupacionais, 28 eram trabalhadores de saúde que se acidentaram com instrumentos perfurocortantes. Este estudo mostra uma problemática mais complexa ao se verificar que a força de trabalho em saúde nos Estados Unidos é composta por 6 milhões de trabalhadores ${ }^{(15)}$.

Um estudo realizado no Rio de Janeiro analisou os acidentes de trabalho ocorridos com fluidos biológicos e mostrou que as percepções individuais, coletivas e institucionais indicam reestruturações das normas para as questões de segurança no ambiente hospitalar, visando à minimização destes riscos ${ }^{(16)}$.

A quimioprofilaxia reduz o risco de soroconversão ao vírus HIV, porém o início da terapia medicamentosa deve ser imediata após a exposição a fluídos biológicos. Os comportamentos e as condutas pessoais tornam-se um agravante, pois quando os trabalhadores abandonam a medicação aumentam a vulnerabilidade para uma possível soroconversão(17). Este estudo mostra que 58,3\% dos trabalhadores expostos a material biológico não receberam as orientações necessárias no momento do acidente. O mais significativo e preocupante foi a porcentagem de $42,8 \%$ de trabalhadores que não terminaram a quimioprofilaxia indicada. Somada a esta gravidade, destes, $23,8 \%$ recusaram a medicação prescrita.

Em outro estudo, o percentual de $66,6 \%$ não seguiu corretamente as normas preconizadas. Estes dados são expressivos, o que indica a necessidade de revisão e elaboração de estratégias de prevenção dos acidentes com exposição aos líquidos corporais humanos ${ }^{(18)}$. As estraté- 
gias preventivas podem reduzir ou minimizar a exposição biológica. Estas estratégias incluem: a imunização para o HBV, o uso de barreiras de proteção e a profilaxia medicamentosa pós-exposiçao ao HIV, quando indicada(14).

Essa exposição ocupacional é vivenciada no cotidiano do trabalho, porém existe um grande número de subnotificação desses acidentes e no protocolo de monitoramento biológico, constatando que muitas vezes os trabalhadores não aderem ao monitoramento ${ }^{(19)}$.

Assim, considerando a alta frequência e a gravidade da exposição ocupacional com fluídos biológicos e a necessidade de disseminar conhecimentos sobre as práticas preventivas, pretende-se, com este texto, fornecer subsídios para as condições de trabalho segura, no que diz respeito a exposição dos trabalhadores.

Este texto relata a vivência na Unidade Saúde do Trabalhador, que é referência na cidade de Curitiba para o atendimento dos trabalhadores expostos aos fluidos biológicos. As orientações aqui relatadas estão respaldadas na legislação e são transmitidas durante o acompanhamento por enfermeiros, médico infectologista, psicólogo, assistente social, docentes do Grupo de estudo Multidisciplinar de Saúde do Adulto (GEMSA) da Universidade Federal do Paraná e docentes Grupo de Pesquisa de Qualidade de Vida do Departamento ENO da EEUSP. Propõe-se, portanto, construir um guia que contribua para a adesão ao protocolo de monitoramento biológico, segundo as orientações e intervenções a seguir.

\section{Informações aos trabalhadores}

- Mesmo se a exposição for uma gota na mucosa, o trabalhador deve realizar o acompanhamento: Independente do tipo da exposição e quantidade de secreção, o acompanhamento deve ser realizado durante 6 meses ${ }^{(7)}$.

- Se o paciente-fonte for criança ou idoso, existe o risco de contaminação: Para qualquer paciente-fonte, independente de sua idade, condição clínica, opção celibatária ou orientação sexual, devem ser solicitadas sorologias para HIV, HBV e HCV. O risco de contaminação está presente ${ }^{(7)}$.

- Resultado negativo de HIV após teste no paciente-fonte: Entre os agentes transmitidos pelo sangue, o menos infectante deles é o HIV. Os vírus das Hepatites B e $\mathrm{C}$ apresentam transmissibilidade mais elevada que o HIV e não podem, em hipótese nenhuma, serem negligenciados na avaliação do trabalhador exposto. Pacientes com exposição recente ao HIV, há menos de três meses podem estar em janela imunológica e com teste negativo. Portanto, é necessário investigar se o paciente-fonte esteve exposto à infecção nos últimos três meses. Se for o caso, repetir os exames. Se o paciente-fonte for negativo com relação ao HIV, não está indicado a quimioprofilaxia anti-retroviral, mas deverá ser feito o acompanhamento sorológico tanto para HIV quanto para as Hepatites B e C, sendo a possibilidade de infecção para HIV baixíssima ${ }^{(7)}$.
- Todos os profissionais de saúde devem tomar a vacina contra Hepatite B: A vacina para Hepatite B está disponível no Sistema Único de Saúde para todos os profissionais de saúde, sendo a imunização realizada através de três doses, com intervalo de um mês entre a primeira e a segunda dose e de seis meses entre a primeira e a terceira dose $(0,1 \text { e } 6 \text { meses })^{(7)}$.

- Resultados do exame realizado no dia da exposição ocupacional: $\mathrm{O}$ exame realizado no dia da exposição biológica serve como segurança ao trabalhador para comprovar seu estado de saúde no momento da exposição biológica, sendo necessário repetir o exame para HIV com 30 dias, devido à janela imunológica. O período total para detecção de anticorpos, isto é, a janela imunológica, é caracterizada por marcadores virais indetectáveis em amostra de sangue ${ }^{(20)}$.

- Doação de sangue após a exposição biológica: Nos casos de acidente com material biológico, deve-se evitar doação de sangue no período de até 12 meses $^{(7)}$.

- Gravidez após a exposição ocupacional: Recomenda-se não engravidar durante esse período, o trabalhador exposto deverá utilizar preservativo em todas as relações sexuais, principalmente nas 6 a 12 semanas pós-exposição, apenas por segurança(7).

- Amamentação após a exposição ocupacional: O médico e o profissional enfermeiro que assistir o acidentado deverá orientar que o aleitamento materno deve ser interrompido pós-exposição ${ }^{(7)}$.

- Recusa da realização dos exames por parte do paciente-fonte: Os exames do paciente-fonte só poderão ser realizados caso ele permita. Não existe nenhuma lei que obrigue a realizar os exames. Nestes casos, o médico assistente deve ser contactado e solicitado para que o mesmo esclareça ao paciente sobre a importância dos exames ${ }^{(7)}$.

\section{Conduta frente à exposição biológica}

- Ações que devem ser tomadas imediatamente após o acidente com fluídos biológicos: Orientar o trabalhador a manter a calma; orientar a lavagem do local do ferimento com água e sabão nos casos de exposição percutânea ou cutânea; orientar a lavagem abundantemente com água ou solução fisiológica as exposições em mucosas; acionar o serviço no qual o trabalhador sofreu a exposição ocupacional, para providenciar os exames sorológicos para Hepatite $\mathrm{B}$, Hepatite $\mathrm{C}$ e se possível teste rápido para HIV quando o paciente fonte for conhecido. O trabalhador deve procurar atendimento emergencial no Pronto Socorro preferencialmente de 2 a 6 horas após o acidente, para realizar os exames de Determinante Antigênico encontrado na Superfície do HBV (HBsAg), Anticorpo contra o Antígeno do core do Vírus da Hepatite B (Anti HBc), Anticorpo contra Vírus da Hepatite C (Anti HCV) e Anticorpo contra o Virus da Imunodeficiência Humana (Anti HIV) e ver a necessidade do uso da quimioprofilaxia. A indicação da quimioprofilaxia será de acordo com a conduta médi- 
ca no Pronto Socorro, conforme protocolo do Ministério da Saúde; orientar o trabalhador para que seja emitida a Comunicação de Acidente de Trabalho (CAT) pela empresa e que o preenchimento do campo do atestado médico seja realizado no Pronto Socorro. Orientar o trabalhador na realização do acompanhamento por 6 meses após a exposição biológica; reforçar que o abandono no monitoramento pode comprometer sua saúde, uma vez que a não adesão é em torno de $70 \%$. O trabalhador deverá informar à chefia imediatamente se apresentar os seguintes sintomas: linfodenopatia, rash, dor de garganta e sintomas de gripe, que são sintomas sugestivos de soroconversão aguda. Orientar a suspensão da amamentação temporária, e da intenção de gravidez, bem como a suspensão da doação de sangue nesse período quando o trabalhador for doador e reforçar ao trabalhador as medidas de biossegurança e precauções básicas em serviço ${ }^{(7,8)}$.

\section{Conduta após a exposição biológica}

- Todos os trabalhadores deverão realizar o acompanhamento e monitoramento durante 0 período de 6 meses: Todos os trabalhadores após a exposição ocupacional devem, de acordo com o protocolo do Ministério da Saúde, realizar o acompanhamento durante 6 meses, independente do tipo de exposição ${ }^{(7)}$.

- Profilaxia após exposição biológica: Só farão uso de profilaxia os trabalhadores que obtiverem o resultado positivo para HIV e Hepatite B do paciente-fonte, ou se este paciente-fonte tiver uma história clínico-epidemiológica sugestiva para infecção pelo HIV e Hepatite B. Ou ainda, se o paciente-fonte for desconhecido, só terá indicação à quimioprofilaxia, dependendo da gravidade do acidente (maior volume de sangue, lesões profundas, agulhas de grosso calibre, entre outros) e do perfil epidemiológico do local de trabalho. A Profilaxia Pós-Exposição (PPE), quando indicada para o HIV, deverá ser iniciada o mais rápido possível, preferencialmente, nas primeiras duas horas após acidente. Recomenda-se que o prazo máximo seja de até 72 horas após o acidente. A duração para quimioprofilaxia é de 28 dias.

Os esquemas preferenciais para PPE estabelecidos pelo Ministério da Saúde (MS) são: básico - Zidoduvina (AZT)+Lamiduvina (3TC), preferencialmente combinados em um mesmo comprimido; expandido preferencial em que são combinados três antirretrovirais ou dois antirretrovirais mais um inibidor da protease. O mais indicado é AZT+3TC+TENOFOVIR (TDF), pois oferece como vantagem a comodidade posológica (três comprimidos ao dia), maior adesão e menor toxicidade em curto prazo; outra opção é o AZT+3TC+lopinavir/ritonavir (LPV/r) e expandido alternativo, recomendado em casos de intolerância ou efeitos adversos ao esquema preferencial, sendo $\mathrm{TDF}+3 \mathrm{TC}+\mathrm{LPV} / \mathrm{r}^{(20)}$

A profilaxia para Hepatite $B$ para os expostos que não foram vacinados é a imunoglobulina contra Hepatite $B$, num período de sete dias após a exposição ocupacional mas, idealmente, nas primeiras 24 horas após a exposição(20).

\section{Condutas preventivas frente à exposição biológica}

- Materiais Perfurocortantes: não retirar, dobrar ou reencapar agulhas já utilizadas em procedimentos. Tomar cuidado e ter atenção com material perfurocortante como lâminas, vidros e bisel de catetes curtos, procurando descartá-los em recipientes adequados para esta finalidade, de acordo com as normas vigentes.

- Lavagem das mãos: após a realização de procedimentos que envolvam presença de sangue, fluidos corpóreos, secreções e itens contaminados por estes ou após a retirada das luvas; antes e após contato com cada paciente.

- Equipamento de Proteção Individual: utilizar luvas descartáveis quando o procedimento ou situação envolver a possibilidade de contato com material biológico como sangue, líquor, sêmen, secreção vaginal, membranas mucosas, pele não íntegra e qualquer objeto que possa estar contaminado. As máscaras, óculos protetores e protetor de face devem ser utilizados nas situações em que possa ocorrer contato através de respingos ou esguicho de sangue e secreções. Utilizar avental indicado para cada situação, sempre que houver possibilidade de ocorrer contaminação por material biológico. Os calçados devem ser fechados sempre, e são indicadas botas impermeáveis quando houver a possibilidade de contato dos pés com ambientes úmidos ou molhados.

- Medidas Preventivas: utilizar material de apoio como cuba-rim e bandeja, durante qualquer procedimento invasivo, para colocação de material perfurocortante. Separar com muita atenção os materiais perfurocortantes dos campos e roupas utilizadas em procedimentos invasivos, após a realização dos mesmos. Jamais colocar materiais perfurocortantes nos lixos comuns dos quartos, corredores e postos. A caixa de perfurocortante deverá ser lacrada e transportada pelas alças mantendo-a afastada do corpo. Não guardar este material nos bolsos ou lençóis ${ }^{(9)}$.

\section{CONCLUSÃO}

Os acidentes de trabalho com fluidos biológicos em trabalhadores de saúde são os mais frequentes dentre os acidentes e os mais graves, podendo causar o desenvolvimento de doenças. A exposição ocupacional entre estes, mais especificamente, entre os da enfermagem, pode ser atribuída a vários fatores direta ou indiretamente, tais como: a assistência integral e direta ao paciente, administrando medicação e realizando curativos; limpeza e esterilização de materiais cirúrgicos e instrumentais diversos, carga excessiva de trabalho; condições impróprias da realização do processo de trabalho; a não-adoção das medidas preventivas, entre elas o uso dos equipamentos de proteção individual, e a não-vacinação contra hepatite B; entre outros.

Assim, podemos afirmar que para modificação do quadro exposto acima é necessário orientar os trabalhadores, principalmente porque a exposição aos fluidos biológicos os submete a vários problemas de saúde, com consequên- 
cias muitas vezes nefastas e irreversíveis, podendo gerar o afastamento, o desemprego e a impossibilidade de retorno ao mesmo posto de trabalho.

Faz-se necessário também o seu monitoramento, através do protocolo estabelecido pelo Ministério da Saúde e sua sensibilização à adesão. Verificamos que, mesmo com esse protocolo, que deve ser seguido por todos os

\section{REFERÊNCIAS}

1. Balsamo AC, Felli VEA. Estudo sobre acidentes de trabalho com exposição aos líquidos corporais humanos em trabalhadores da saúde de um hospital universitário. Rev Latino Am Enferm. 2006;14(3):346-53.

2. Caetano JA, Soares, E, Braquehais, AR, Rolim, KAC. Acidentes de trabalho com material biológico no cotidiano da enfermagem em unidade de alta complexidade. Enferm Global. 2006;9(1):1-10.

3. Sarquis LMM. O monitoramento do trabalhador de saúde, após exposição a fluidos biológicos [tese doutorado]. São Paulo: Escola de Enfermagem, Universidade de São Paulo; 2007.

4. Sarquis LMM. Acidentes de trabalho com instrumentos perfurocortantes: ocorrência entre os trabalhadores de enfermagem [dissertação]. São Paulo: Escola de Enfermagem, Universidade de São Paulo; 1999.

5. Marziale MHP, Robazzi MLCC. Accidentes de trabajo con material corto punzante en enfermeras de hospitales. Nure Investig. 2004;2(1):1-7.

6. Sarquis LMM, Felli VEA. Acidentes de trabalho com instrumentos perfurocortantes entre os trabalhadores entre os trabalhadores de enfermagem. Rev Esc Enferm USP. 2002;36(3): 222-30.

7. Brasil. Ministério da Saúde. Secretaria de Atenção à Saúde. Departamento de Ações Programáticas Estratégicas. Exposição a materiais biológicos. Brasília; 2006.

8. Sarquis LMM, Felli VEA, Miranda FMA, Guimarães HV, Oliveira GP. A adesão ao protocolo de monitoramento dos trabalhadores de saúde após a exposição a fluidos biológicos: uma problemática vivenciada em um ambulatório de saúde do trabalhador. Cogitare Enferm. 2005;10(2):47-53.

9. Centers for Disease Control and Prevention (CDC). Department of Health and Human Services. Sharps injury prevention program workbook [Internet]. Atlanta; 2007 [cited 2010 Ago 10]. Available from: 26/08/10http://www.cdc.gov/sharpssafety/pdf/sharpsworkbook_2008.pdf

10. Lacerda RA. Exposição ocupacional ao sangue e outras substâncias orgânicas de pacientes em unidades de centro cirúrgico de hospitais do Brasil [tese doutorado]. São Paulo: Escola de Enfermagem, Universidade de São Paulo; 2000. trabalhadores de saúde, a realidade vivenciada mostra a não-adesão devido ao seu desconhecimento, pela dificuldade de mudanças de comportamento e pela falta de investimentos institucionais em treinamentos dos seus trabalhadores. Nesse sentido, o conteúdo abordado neste texto constitui um guia para subsidiar as intervenções institucionais necessárias para a valorização do profissional de saúde em seus aspectos bio-psicossociais.

11. Tomazin CC, Benatti MCC. Acidente do trabalho por material perfurocortante em trabalhadores de enfermagem. Rev Gaúcha Enferm. 2001;22(2):60-73.

12. Marziale $\mathrm{MHP}$, Rodrigues $\mathrm{CM}$. A produção científica sobre os acidentes de trabalho com material perfurocortante entre trabalhadores de enfermagem. Rev Latino Am Enferm. 2002;10(4):571-7.

13. Sêcco IAO, Robazzi MLCC, Gutierrez PR, Matsuo T. Acidentes de trabalho e riscos ocupacionais no dia-a-dia do trabalhador hospitalar: um desafio para a saúde do trabalhador. Rev Espaço Saúde. 2002;4(1):19-24

14. Pruss-Ustun A, Rapiti E, Hutin Y. Sharps injuries: global burden of disease from sharps injuries to health-care workers. Geneva: Word Health Organization; 2003 (WHO Enviromental Burden of Disease Series, 3).

15. Sepkpwitz KA, Eisenbergt L. Occupational deaths among healthcare workers. Emerg Infect. Dis. 2005;11(7):1003-8.

16. Neves SMFM. Estudo dos acidentes de trabalho com material biológico contaminado entre profissionais de saúde no Centro de Pesquisa Hospital Evandro Chagas/Fundação Oswaldo Cruz [dissertação]. Rio de Janeiro: Universidade do Estado do Rio de Janeiro; 2000

17. Mariano CGG, El-Far F, Wey SB, Medeiros EAS. Cut and puncture accidents involving health care workers exposed to biological materials. Braz J Infect Dis. 2001;5(5):235-42.

18. Balsamo AC. Estudo sobre os acidentes de trabalho com exposição aos líquidos corporais humanos em trabalhadores de saúde [dissertação]. São Paulo: Escola de Enfermagem, Universidade de São Paulo; 2002.

19. Sarquis LMM, Felli VEA Recomendações em saúde aos trabalhadores expostos a fluidos biológicos. REME Rev Min Enferm. 2008;12(3):331-88.

20. Brasil. Ministério da Saúde. Secretaria de Vigilância em Saúde. Departamento de DST, Aids e Hepatites Virais. Recomendações para terapia anti-retroviral em adultos infectados pelo HIV: suplemento III tratamento e prevenção [Internet]. Brasília; 2008 [citado 2010 dez. 20]. Disponível em: http:// www.crt.saude.sp.gov.br/resources/crt_aids/pdfs/guia_de_ tratamento_pos_exposicao.pdf
Correspondência: Fernanda Moura D'Almeida Miranda Av. República Argentina, 4406 CEP 81050-000 - Curitiba, PR, Brasil 